\title{
Levothyroxine Replacement for Primary Hypothyroidism Can Be Given Between Meals with Similar Effectiveness at Various Times of the Day
}

\section{Charles H. Emerson}

Skelin M, Lucijani T, Liberati-Cizmek AM, Klobucar SM, Lucijanic M, Jakupovic L, Bakula M, Loncar JV, Marusic S, Matic T, Romic Z, Dumic J, Rahelic D 2018 Effect of timing of levothyroxine administration on the treatment of hypothyroidism: a three-period crossover randomized study. Endocrine. Epub 2018 Jul 24. PMID: 30043093.

\section{SUMMARY}

\section{Background}

The American Thyroid Association (ATA) guidelines (1) recommend levothyroxine $\left(\mathrm{L}-\mathrm{T}_{4}\right)$ replacement for treatment of primary hypothyroidism. After its administration, a fraction of the dose is converted to L-triiodothyronine $\left(\mathrm{T}_{3}\right)$. The thyroid gland secretes both $\mathrm{T}_{4}$ and $\mathrm{T}_{3}$, and a large proportion of the circulating $\mathrm{T}_{3}$ is derived from conversion of secreted $\mathrm{T}_{4}$ to $\mathrm{T}_{3}$. Therefore, it would be ideal to treat hypothyroidism by a method that reproduces all aspects of $\mathrm{T}_{4}$ and $\mathrm{T}_{3}$ production and degradation, including intricately regulated aspects of thyroid hormone secretion and peripheral $\mathrm{T}_{3}$ genesis that are initiated by hypothalamic circadian rhythms, feedback loops, nutritional status, and stress responses.

Most patients with hypothyroidism are treated with tablets containing $\mathrm{T}_{4}$ or $\mathrm{T}_{4} / \mathrm{T}_{3}$ combinations. A limitation of this treatment is less-than-complete gastrointestinal absorption of the active dose, particularly when taken with meals. Although it is generally agreed that thyroid hormone tablets are best not taken with meals, there are only a few studies and brief reports supporting this idea, some of which are cited here (2-6). In the study reviewed here, Skelin et al. (2) compared the effectiveness of levothyroxine tablet dosing at three different times of the day.

\section{Methods}

Adult patients ( $>17$ years) with primary hypothyroidism who were taking a stable generic dose of

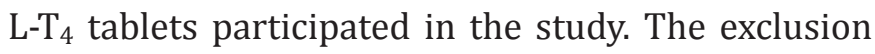
criteria were pregnancy, Addison's disease, and celiac disease. Patients were also excluded if, in the opinion of their physician, there was a need to change their L-T $\mathrm{T}_{4}$ dose.

This was an open-label, crossover study with each of the three 8-week periods being distinguished by the time of the day when patients took a daily dose of $\mathrm{L}-\mathrm{T}_{4}$. One time was a half hour before breakfast (BEFORE-BREAKFAST), another was an hour before the main meal of the day (BEFORE-MAINMEAL) and the third at bedtime (BEDTIME), at least 2 hours after the last meal of the day. The sequence of the schedule for each patient was assigned in a randomized fashion. The study was performed in four hospitals in Croatia.

Medical examinations and laboratory tests were obtained at the beginning and end of each treatment schedule. Prior to each period, patients received detailed counseling regarding their upcoming $\mathrm{L}^{-} \mathrm{T}_{4}$ treatment schedule. Decisions to exclude patients from the study based on the assessment of their physicians were also made at this time. The end points of the study 


\section{Various Times of the Day}

were serum levels of $\mathrm{TSH}, \mathrm{FT}_{3}, \mathrm{FT}_{4}$, triglycerides, $\mathrm{HDL}$ cholesterol, LDL cholesterol, and total cholesterol.

\section{Results}

A total of 84 patients, 9 men and 75 women, completed the study. Additional exclusions consisted of 13 patients who did not finish all three dose schedules, 4 whose levothyroxine dose was changed, and 3 because of protocol violations. As the data were not normally distributed, nonparametric tests were used for all analyses.

Men and women were of similar age (interquartile range [IQR], 45-68 years for women and 24-63 for men; $\mathrm{P}=0.51$ ) and body-mass index (mean BMI, 28.3 for women and 26.7 for men; $\mathrm{P}=0.40$ ). About $20 \%$ of the patients were taking statins, about $10 \%$ were taking proton-pump inhibitor drugs or H2-receptor antagonists, and a slightly higher percentage were taking antipsychotic medications. All but about $5 \%$ of patients had been taking $\mathrm{L}^{-} \mathrm{T}_{4}$ for at least 1 year, and almost a quarter of patients had been taking $\mathrm{L}-\mathrm{T}_{4}$ for more than 10 years.

Baseline serum thyroid-function tests were a median of $2.0 \mathrm{mIU} / \mathrm{L}$ for serum TSH (IQR, 1.2-2.8), 15.6 $\mathrm{pmol} / \mathrm{L}$ for $\mathrm{FT}_{4}$ (IQR, 13.6-17.3), and $4.5 \mathrm{pmol} / \mathrm{L}$ for $\mathrm{FT}_{3}$ (IQR, 4.1-5.0). Median serum TSH concentrations while on the BEFORE-BREAKFAST schedule were $1.9 \mathrm{mIU} / \mathrm{L}$ (IQR, 1.1-2.8),on the BEFORE-MAINMEAL schedule $2.3 \mathrm{mIU} / \mathrm{L}$ (IQR, 1.4-3.3), and on the BEDTIME schedule $2.2 \mathrm{mIU} / \mathrm{L}$ (IQR, 1.3-3.4); none of these were significantly different from the others by the Friedman ANOVA test for repeated measures. Similarly, serum $\mathrm{FT}_{4}$ and $\mathrm{FT}_{3}$ concentrations did not differ among the values at baseline and at the end of the BEFORE-BREAKFAST, BEFORE-MAINMEAL, and BEDTIME schedules. As was the case for serum $\mathrm{TSH}, \mathrm{FT}_{4}$, and $\mathrm{FT}_{3}$, there was no significant difference between serum triglycerides, LDL cholesterol, HDL cholesterol, or total cholesterol among values at baseline or at the end of any of the three $\mathrm{L}^{-} \mathrm{T}_{4}$ treatment schedules.

Various subgroup analyses were performed, including patients $>65$ years, those taking and not taking statins (or omega-3 acids or fenofibrate), those taking and not taking proton-pump inhibitors or H2-receptor antagonists, or those with BMIs $\geq 30$ and $<30$. These yielded similar values for the three treatment schedules except that serum HDL cholesterol was significantly lower (P $=0.027)$ and serum triglycerides were significantly higher $(\mathrm{P}=0.041)$ at the end of the BEDTIME schedule in patients with BMI values of less than 30 .

\section{Conclusions}

Serum TSH and thyroid hormone concentrations are similar in patients with primary hypothyroidism on the same replacement doses of $\mathrm{L}^{-} \mathrm{T}_{4}$ when this treatment is taken 30 minutes before breakfast, an hour before the main meal of the day, or at bedtime, provided that it is taken at least 2 hours after the last meal of the day. Serum lipid/cholesterol profiles are also similar, but more definitive studies are needed to determine if there is an interaction between BMI and the efficacy of the different $\mathrm{L}-\mathrm{T}_{4}$ treatment schedules.

\section{ANALYSIS AND COMMENTARY}

In its 2014 guidelines (1), the ATA recommended that $\mathrm{L}-\mathrm{T}_{4}$ be taken either 60 minutes before breakfast or at bedtime, at least 3 hours after the evening meal.
The Skelin et al. study did not examine these recommendations precisely, since their $\mathrm{L}^{-\mathrm{T}_{4}}$ administration times were 30 minutes before breakfast or at least 2 


\section{Can Be Given Between Meals with Similar Effectiveness at Various Times of the Day}

hours before bedtime, but the BEFORE-BREAKFAST and BEDTIME schedules were roughly comparable. In addition, Skelin et al. (2) added a third time, BEFORE-MAINMEAL. As implied in the last paragraph of their paper this time was probably before the midday meal, but further clarification on this point was not provided.

Skelin et al. indicated that this was the first randomized crossover study to directly compare all three previously investigated times of levothyroxine administration. Unlike some earlier studies, the equilibration period for the study, 8 weeks, was also longer. In contrast to some earlier reports, evidence for increased $\mathrm{L}-\mathrm{T}_{4}$ absorption at bedtime (3) was not noted.

The strengths of the study reviewed here (2) are its prospective nature and crossover randomized design. Among its limitations was the likelihood that the subgroup analyses were underpowered and affected by type I error. Even though the authors took steps to avoid the latter problem, they expressed doubt about the validity of an interaction of BMI with thyroid-function test data. Another limitation was that patient compliance was not measured. This is a problem with many studies of this nature. Since the study did not compare two treatments, its open-label nature is not a clear limitation; in fact, the authors considered an alternative blinded regimen of placebo/L- $\mathrm{T}_{4}$ three times a day but rejected this because its demanding nature would bias the study in other ways.

There are at least two areas that future studies in this field need to address. One is to establish definitively those thyroid hormone preparations whose gastrointestinal absorption is not affected by foods, supplements, and medications. The other is to determine, in a crossover trial with a relatively large number of patients, the period before and after meals that impairs $\mathrm{L}-\mathrm{T}_{4}$ absorption. This information should be invaluable for devising better times for patients with hypothyroidism to take their thyroid hormone medication.

It is now well accepted that replacement doses of $\mathrm{L}^{-\mathrm{T}_{4}}$ tablets should not be taken with meals unless this is the only reliable way the patient can adhere to a daily schedule for taking thyroid hormones. Above all, patients in whom thyroid hormone therapy is indicated for hypothyroidism should be counseled as to the overriding importance of taking this medication and maintaining regular medical appointments. Given the many differences in preferences and lifestyles among patients, the major contribution of the Skelin et al. study is that it provides more choices for patients as to when to take their replacement dose of levothyroxine. Discussions with patients regarding this is an important aspect of the management of primary hypothyroidism.

\section{References}

1. Jonklaas J, Bianco AC, Bauer AJ, Burman KD, Cappola AR, Celi FS, Cooper DS, Kim BW, Peeters RP, Rosenthal S, Sawka AM 2014 Guidelines for the treatment of hypothyroidism: prepared by

the American Thyroid Association Task Force on Thyroid Hormone Replacement. Thyroid 24:1670-1751. 
Levothyroxine Replacement for Primary Hypothyroidism

Charles H. Emerson

Can Be Given Between Meals with Similar Effectiveness at

Various Times of the Day

2. Skelin M, Lucijani T, Liberati-Cizmek AM, Klobucar SM, Lucijanic M, Jakupovic L, Bakula M, Loncar JV, Marusic S, Matic T, Romic Z, Dumic J, Rahelic D 2018 Effect of timing of levothyroxine administration on the treatment of hypothyroidism: a three-period crossover randomized study. Endocrine. Epub 2018 Jul 24.

3. Benvenga S, Bartolone L, Squadrito S, Lo Giudice F, Trimarchi F 1995 Delayed intestinal absorption of levothyroxine. Thyroid 5:249-253.

4. Bolk N, Visser TJ, Kalsbeek A, van Domburg RT, Berghout A 2007 Effects of evening vs morning thyroxine ingestion on serum thyroid hormone profiles in hypothyroid patients. Clin Endocrinol (Oxf) 66:43-48.
5. Bach-Huynh TG, Nayak B, Loh J, Soldin S, Jonklaas J 2009 Timing of levothyroxine administration affects serum thyrotropin concentration. J Clin Endocrinol Metab 94:3905-3912. Epub 2009 Jul 7.

6. Ala S, Akha O, Kashi Z, Asgari H, Bahar A, Sasanpour N 2015 Dose administration time from before breakfast to before dinner affect thyroid hormone levels? Caspian J Intern Med 6:134-140.

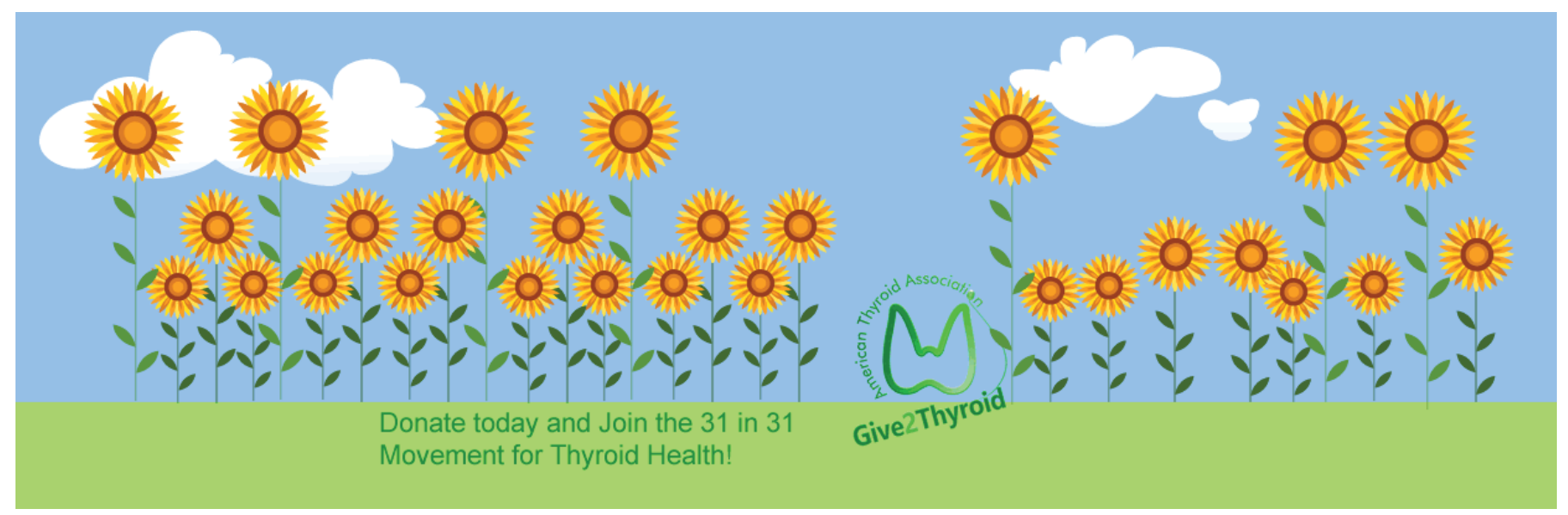

